\section{Cytomegalovirus-induced Colon Perforation in Systemic Lupus Erythematosus}

\section{To the Editor:}

Acute abdomen has been reported in $10 \%$ of patients with systemic lupus erythematosus (SLE) ${ }^{1}$. The differential diagnoses of abdominal pain in SLE do not differ significantly from those in patients without SLE with the exception of serositis, thrombotic, or hemorrhagic events associated with antiphospholipid antibodies, mesenteric vasculitis (MV), and cytomegalovirus (CMV) infection. The distinction between MV and CMV infection is critical because both are life-threatening disorders requiring radically different therapeutic approaches. We describe the case of a patient with SLE who was profoundly immunosuppressed and who presented with an acute surgical abdomen secondary to a colonic perforation, and emphasize the relevance of considering a viral etiology in this setting.

A 54-year-old woman with a 22-year history of SLE presented to the Emergency Department with acute abdominal pain. Previous manifestations of her disease included serositis, arthritis, nephritis, and cytopenias. In 2001, the patient received induction (cyclophosphamide) and maintenance [azathioprine (AZA)] therapy for class IV lupus nephritis (National Institutes of Health criteria activity index 5 , chronicity index 8). Optimization of the AZA dose was limited because of myelosuppression. Over the previous 8 months, her creatinine had increased progressively from a baseline of 200 $\mathrm{mmol} / \mathrm{l}$ to $350 \mathrm{mmol} / \mathrm{l}$. A month prior to the current admission, the patient had a preemptive renal transplant evaluation. At the time, prednisone (50 $\mathrm{mg}$ /day) was added to her chronic immunosuppressive therapy (AZA $50 \mathrm{mg} /$ day).

On admission, the patient had excruciating, stabbing, and diffuse abdominal pain. She denied having nausea, vomiting, dysphagia, diarrhea, melena, weight loss, urinary symptoms, or fever. On examination, her abdomen was rigid and diffusely tender, with guarding in all quadrants and rebound tenderness. Results of laboratory tests included leukocytes $1.8 \times$ $10^{9} / 1$, absolute lymphocytes $0.10 \times 10^{9} / 1$, hemoglobin $11.7 \mathrm{~g} / \mathrm{dl}$, platelets $139 \times 10^{9} / 1$, alanine aminotransferase $64 \mathrm{U} / 1$, aspartate aminotransferase 48 U/1, alkaline phosphatase $88 \mathrm{U} / 1$ (normal 42-98), amylase $442 \mathrm{U} / 1$ (normal 20-130), lipase $65 \mathrm{U} / 1$ (normal 14-45), creatinine $433 \mathrm{umol} / 1$, complement fraction 3 (C3) $0.77 \mathrm{~g} / 1$ (normal $0.79-1.52$ ), and C4 $0.33 \mathrm{~g} / 1$ (normal $0.16-0.38)$. Antinuclear antibody was positive (1:160) with a speckled pattern, while anti-DNA, anti-SM, Ro/La, anticardiolipin antibodies, and lupus anticoagulant were all negative. The SLE Disease Activity Index (SLEDAI) score was 4. An abdominal computed tomography (CT) scan showed free air in the upper retroperitoneal area with an inflammatory focus adjacent to the splenic flexure (Figure 1). A perforation of the distal transverse colon/splenic flexure was diagnosed, for which she underwent resection with end colostomy. On histology, a transmural ulceration of the colon without evidence of vasculitis was found. CMV inclusions were documented adjacent to the perforation by immunohistochemistry (Figure 2). CMV viral load (quantitative PCR) was $1.63 \times 10^{4}$ copies $/ \mathrm{ml}$. Following the diagnosis of CMV gastrointestinal (GI) disease, ophthalmology confirmed the presence of lesions close to retinal vessels consistent with $\mathrm{CMV}$-associated retinitis. AZA was discontinued and ganciclovir was initiated (70 mg intravenous, 3 times weekly). The postoperative course was complicated by venous thrombosis of the right basilic and right peroneal veins, an event that has been associated with CMV infection ${ }^{2}$.

Differential diagnoses of life-threatening abdominal complications in SLE include lupus MV, intestinal pseudo-obstruction, and generalized megaviscera, acute pancreatitis (secondary to SLE or drug-related, i.e., AZA), protein-losing gastroenteropathy, and other less common entities (i.e., eosinophilic enteritis, pneumatosis cystoides intestinalis) $)^{3}$.

MV is the most commonly reported cause of bowel perforation in $\mathrm{SLE}^{1,4}$. MV is a life-threatening disorder with an associated mortality of up to $50 \%$. It occurs in patients with high disease activity (SLEDAI > 8) and usually manifests with insidious diffuse abdominal pain that may be intermittent for months prior to the development of an acute abdomen. The presence of cutaneous vasculitis, central nervous system involvement, thrombocytopenia, lymphopenia, and rheumatoid factor positivity in a patient with SLE with an acute abdomen suggest the diagnosis of $\mathrm{MV}^{1,4,5}$. Common but nonspecific CT findings in patients with MV include dilated bowel, focal or diffuse bowel wall thickening/enhancement (double halo or target sign), mesenteric edema, engorgement of mesenteric vessels (comb sign), and ascites. Involvement of the duodenum was reported to be highly suggestive of vasculitis ${ }^{4}$.

Overt CMV clinical disease in SLE is rare and is associated with high mortality rates. The risk of CMV infection is higher in patients treated with cyclophosphamide or combined immunosuppressive agents (i.e., AZA/mycophenolate acid and glucocorticoids). Other risk factors associated with life-threatening viral infections in SLE include renal insufficiency, antiphospholipid syndrome, and delayed onset of antiviral therapy ${ }^{6}$. CMV involvement of the GI tract usually manifests as single or multiple ulcerations ("punched out" appearance) and GI bleeding. Colonic perforation because of CMV infection is exceedingly rare ${ }^{7}$. The diagnosis of CMV GI disease relies upon culture and histopathology of a tissue biopsy. Although

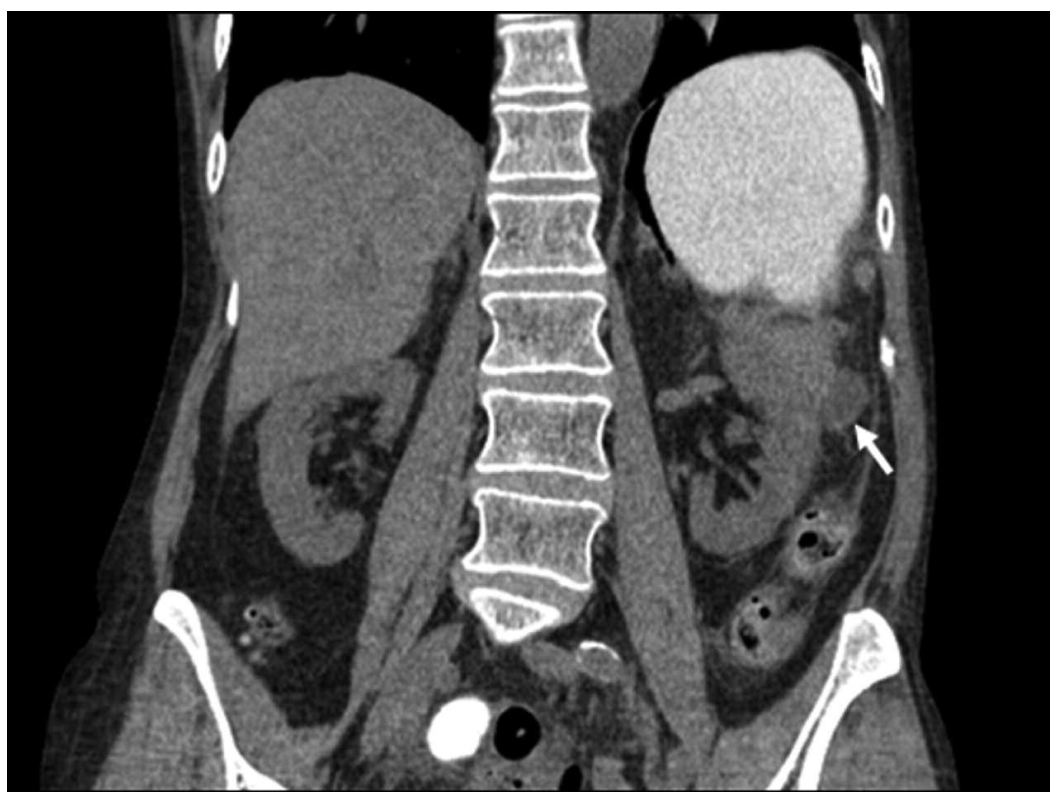

Figure 1. Abdominal computed tomography (coronal image): inflammatory focus adjacent to the splenic colonic flexure (arrow). 


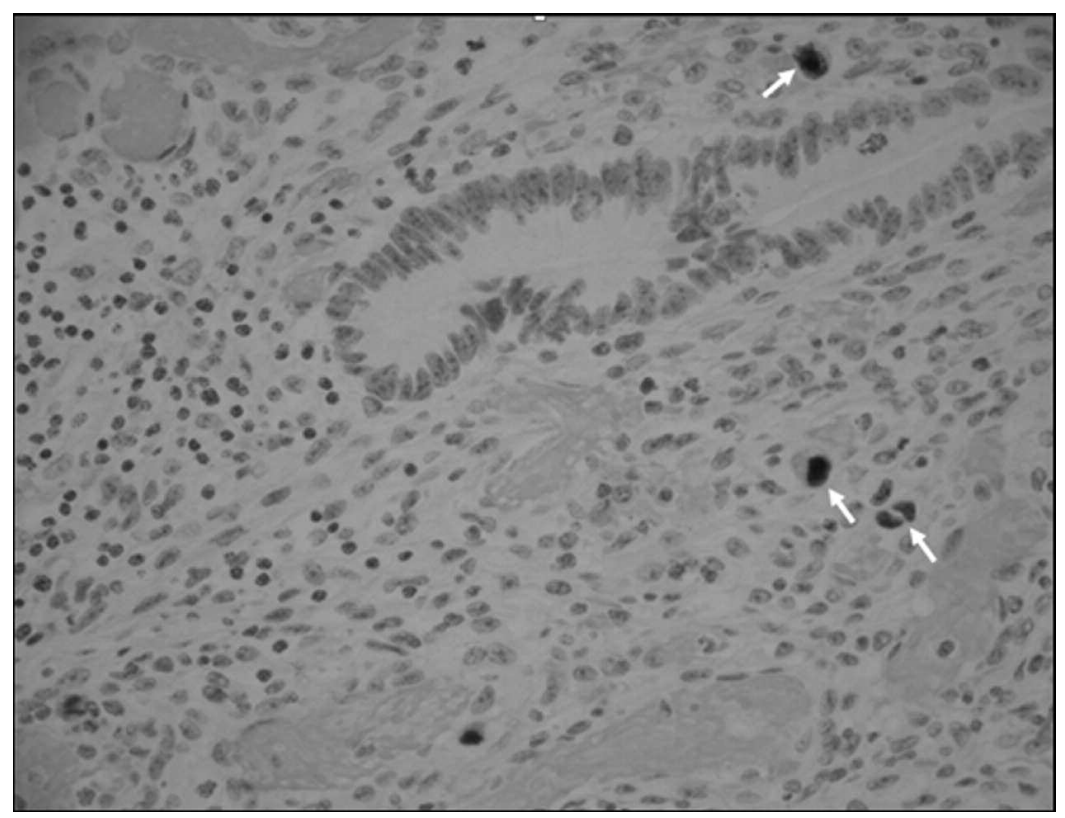

Figure 2. Immunohistochemistry staining: cytomegalovirus inclusions within the granulation tissue adjacent to the colonic perforation (arrows).

the value of CMV DNA tests to establish symptomatic infection in patients with SLE has not been established, the level of CMV load and its rate of increase predict symptomatic disease in transplant patients ${ }^{8}$. Coexisting manifestations such as pneumonitis and/or retinitis should suggest the diagnosis of tissue-invasive CMV disease.

This case emphasizes the challenges of the differential diagnosis between MV and CMV GI involvement in a patient presenting with a colonic perforation. It is of utmost clinical importance to include viral infections as a differential diagnosis in patients with immunosuppressed SLE presenting with an acute abdomen. Performing early serological and molecular studies will confirm diagnosis and guide life-saving therapy.

TETIANA GLUSHKO, MD, Division of Rheumatology, Department of Medicine, McGill University Health Centre, Montreal, Quebec, Canada; VICTORIA A. MARCUS, MD FRCPC, Associate Professor, Department of Pathology, McGill University Health Centre, Montreal, Quebec, Canada; INÉS COLMEGNA, MD, Assistant Professor, Division of Rheumatology, Department of Medicine, McGill University Health Centre, Montreal, Quebec, Canada. Address correspondence to Dr. I. Colmegna, Division of Rheumatology, Department of Medicine, McGill University Health Centre, Royal Victoria Hospital, 1001 Décarie Blvd., Bloc E, M2-3238, Montréal, Québec H4A 3J1, Canada. E-mail: ines.colmegna@mcgill.ca

\section{REFERENCES}

1. Zizic TM, Classen JN, Stevens MB. Acute abdominal complications of systemic lupus erythematosus and polyarteritis nodosa. Am J Med 1982;73:525-31.
2. Justo D, Finn T, Atzmony L, Guy N, Steinvil A. Thrombosis associated with acute cytomegalovirus infection: a meta-analysis. Eur J Intern Med 2011;22:195-9.

3. Malaviya AN, Sharma A, Agarwal D, Kapoor S, Garg S, Singh S, et al. Acute abdomen in SLE. Int J Rheum Dis 2011;14:98-104.

4. Ju JH, Min JK, Jung CK, Oh SN, Kwok SK, Kang KY, et al. Lupus mesenteric vasculitis can cause acute abdominal pain in patients with SLE. Nat Rev Rheumatol 2009;5:273-81.

5. Buck AC, Serebro LH, Quinet RJ. Subacute abdominal pain requiring hospitalization in a systemic lupus erythematosus patient: a retrospective analysis and review of the literature. Lupus 2001;10:491-5.

6. Ramos-Casals M, Cuadrado MJ, Alba P, Sanna G, Brito-Zerón P, Bertolaccini $L$, et al. Acute viral infections in patients with systemic lupus erythematosus: description of 23 cases and review of the literature. Medicine 2008;87:311-8.

7. Strasser C, Wolf EM, Kornprat P, Hermann J, Münch A, Langner C. Opportunistic cytomegalovirus infection causing colonic perforation in a patient with systemic lupus erythematosus. Lupus 2012;21:449-51.

8. Razonable RR, Hayden RT. Clinical utility of viral load in management of cytomegalovirus infection after solid organ transplantation. Clin Microbiol Rev 2013;26:703-27.

J Rheumatol 2016;43:2; doi:10.3899/jrheum.150964 